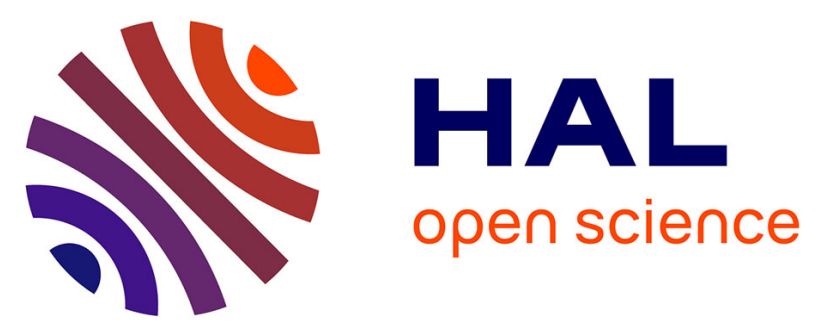

\title{
MAMMALIAN TARGET OF RAPAMYCIN (mTOR) EXPRESSION AND LARYNGEAL SQUAMOUS CELL CARCINOMA PROGNOSIS: NOVEL PRELIMINARY EVIDENCE
}

Gino Marioni, Alberto Staffieri, Luciano Giacomelli, Marco Lionello, Vincenza Guzzardo, Alessandra Busnardo, Stella Blandamura

\section{To cite this version:}

Gino Marioni, Alberto Staffieri, Luciano Giacomelli, Marco Lionello, Vincenza Guzzardo, et al.. MAMMALIAN TARGET OF RAPAMYCIN (mTOR) EXPRESSION AND LARYNGEAL SQUAMOUS CELL CARCINOMA PROGNOSIS: NOVEL PRELIMINARY EVIDENCE. Histopathology, 2011, 58 (7), pp.1148. 10.1111/j.1365-2559.2011.03864.x . hal-00655184

\author{
HAL Id: hal-00655184 \\ https://hal.science/hal-00655184
}

Submitted on 27 Dec 2011

HAL is a multi-disciplinary open access archive for the deposit and dissemination of scientific research documents, whether they are published or not. The documents may come from teaching and research institutions in France or abroad, or from public or private research centers.
L'archive ouverte pluridisciplinaire HAL, est destinée au dépôt et à la diffusion de documents scientifiques de niveau recherche, publiés ou non, émanant des établissements d'enseignement et de recherche français ou étrangers, des laboratoires publics ou privés. 


\section{Histopathology}

\section{MAMMALIAN TARGET OF RAPAMYCIN (MTOR) EXPRESSION AND LARYNGEAL SQUAMOUS CELL CARCINOMA PROGNOSIS: NOVEL PRELIMINARY EVIDENCE}

\begin{tabular}{|r|l|}
\hline Journal: & Histopathology \\
\hline Manuscript ID: & HISTOP-03-10-0183.R1 \\
\hline Wiley - Manuscript type: & Original Article \\
\hline Date Submitted by the \\
Author: & O2-Aug-2010 \\
\hline Complete List of Authors: & $\begin{array}{l}\text { Marioni, Gino; University of Padua, Dept of Medical and Surgical } \\
\text { Specialties } \\
\text { Staffieri, Alberto } \\
\text { Giacomelli, Luciano } \\
\text { Lionello, Marco } \\
\text { Guzzardo, Vincenza } \\
\text { Busnardo, Alessandra } \\
\text { Blandamura, Stella }\end{array}$ \\
\hline Keywords: & \begin{tabular}{l} 
mTOR, laryngeal carcinoma, marker, prognosis, targeted therapy \\
\hline
\end{tabular} \\
\hline
\end{tabular}

\section{SCHOLARONE \\ Manuscripts}


MAMMALIAN TARGET OF RAPAMYCIN (mTOR) EXPRESSION AND LARYNGEAL SQUAMOUS CELL CARCINOMA PROGNOSIS: NOVEL PRELIMINARY EVIDENCE

\author{
Gino Marioni ${ }^{\mathrm{a}}, \mathrm{MD} ;$ Alberto Staffieri ${ }^{\mathrm{a}}, \mathrm{MD}$; \\ Luciano Giacomellib, BD; Marco Lionello ${ }^{\mathrm{a}}$, MD; Vincenza Guzzardo ${ }^{\mathrm{b}}$; \\ Alessandra Busnardo ${ }^{a}$; Stella Blandamura ${ }^{b}, M D$.
}

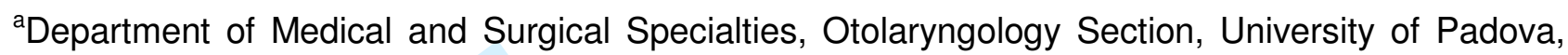
Via Giustiniani 2, 35128 Padova, Italy

${ }^{\mathrm{b}}$ Department of Medical/Diagnostic Sciences and Special Therapies, Anatomic Pathology Section, University of Padova, Via Gabelli 61, 35128 Padova, Italy.

Running title: mTOR in laryngeal SCC

Key words: mTOR, laryngeal carcinoma, marker, prognosis, targeted therapy.

${ }^{*}$ Corresponding author.

Tel.: +39 (0)49 8218626; fax +39 (0)49 8213113 e-mail address gino.marioni@unipd.it (G. Marioni)

The authors declare no conflict of interests. This study was supported in part by grant no. 60A07-4404/09 (G. Marioni) from the University of Padova, Italy ACKNOWLEDGMENTS

The authors thank Frances Coburn for correcting the English version of this paper. 


\begin{abstract}
AIMS Rapamycin has powerful antineoplastic properties against mammalian cells. The mammalian target of rapamycin (mTOR) has a key role in regulating cancer cell proliferation, apoptosis, cell migration and angiogenesis. mTOR pathway activation has been found in several tumor types. This is the first study to assess the relationships between mTOR and clinico-pathological and prognostic parameters in laryngeal squamous cell carcinoma (LSCC).

METHODS/RESULTS mTOR expression was determined in 103 consecutive operable LSCCs. In the mTOR-positive cases, the loco-regional recurrence rate was higher $(p=0.048)$ and the diseasefree survival (DFS) rate shorter $(p=0.031)$ in patients with mTOR expression $>50.7 \%$. In the $N_{0}$ subgroup, the disease recurrence rate was higher $(p=0.034)$ and the DFS shorter $(p=0.009)$ in patients with mTOR expression $>50.7 \%$. In mTOR-positive patients, multivariate analysis found that N-stage $(p=0.0001)$ and mTOR status $(p=0.042)$ were independent indicators of a poor prognosis for LSCC.

CONCLUSIONS mTOR appeared a significant predictor of DFS in univariate and multivariate models. mTOR expression in LSCC may be useful to detect cases at higher risk of recurrence, and $\mathrm{N}_{0}$ patients at higher risk of early loco-regional recurrence who might benefit from more aggressive therapy. The role of modern mTOR inhibitors in multimodality or multi-target strategies against LSCC warrants investigation.
\end{abstract}




\section{INTRODUCTION}

The lipophilic macrolide rapamycin, identified more than 25 years ago during antibiotic screening, has demonstrated potent immunosuppressant and antineoplastic properties against mammalian cells. ${ }^{1}$ The mammalian target of rapamycin (mTOR) is a $290 \mathrm{kDa}$ member of the phosphatidylinositol 3-kinase-related kinase (PIKK) superfamily. mTOR is composed of 2,549 amino acids grouped into highly conserved domains. ${ }^{2} \mathrm{~A}$ rapamycin-binding domain is located centrally, while the kinase domain is located near the carboxyl terminus. ${ }^{3}$

Biochemical investigations have placed mTOR in the growth-factor-activated phosphatidylinositol 3-kinase (PI3K), Akt (protein kinase B) signaling pathway, downstream from Akt. mTOR kinase is also activated by changes that reduce the function of the phosphatase and tensin homolog (PTEN) tumor suppressor protein, or increase the catalytic subunit of PI3K, both of which induce abnormal Akt activation. ${ }^{3}$

Physiologically, mTOR is a central eukaryotic cell growth and proliferation controller and it has a crucial role in regulating important cell functions, such as survival, mobility and angiogenesis. ${ }^{4}$ mTOR activation results in the phosphorylation of several targets downstream. mTOR mediates the translation of mRNAs related to cell cycle check-points and regulates the expression of survival factors, such as c-myc, HIF-1a, and vascular endothelial growth factor (VEGF), as well as being is involved in regulating cyclin A, cyclin-dependent kinases (cdk 1/2), cdk inhibitors ( $\mathrm{p} 21^{\mathrm{Cip} 1}$ and $\mathrm{p} 27^{\mathrm{Kip} 1}$ ), RNA polymerases, protein phosphatases (PP2A, PP4, and PP6) and CLIP170. (Jiang and Liu, 2008) mTOR also has a key role in regulating cancer cell proliferation, apoptosis, cell migration and tumor angiogenesis. ${ }^{5,6}$ mTOR pathway activation and mTOR over-expression have been found in several tumor types, including hepatocellular, renal cell, prostate, and breast cancers ${ }^{4}$. Furthermore, cancer treatment with mTOR inhibitors has produced promising results in various malignancies (breast cancer, prostate cancer, malignant glioma, leukemia, lymphoma, melanoma, and renal cell carcinoma). ${ }^{7}$

The aim of the present study was to investigate mTOR expression in a cohort of 103 consecutive cases of laryngeal squamous cell carcinoma (SCC). This is also the first study to investigate the relationships between the expression of mTOR (an important target of molecular 
therapy) and conventional clinico-pathological and prognostic parameters in laryngeal SCC. Furthermore, in order to investigate the relation between mTOR and survival factors and cdk inhibitors in laryngeal SCC, we assessed by immunohistochemistry c-myc, VEGF and p27 $7^{\text {Kip1 }}$ expressions in the 20 patients with higher mTOR expression and in the 20 patients with lower mTOR expression. 


\section{METHODS}

\section{Patients}

The study was conducted on 103 consecutive cases of laryngeal SCC (92 men and 11 women, with a mean age of $64.4 \pm 9.1$ years). All patients underwent microlaryngoscopy with laryngeal biopsy, upper aerodigestive tract endoscopy, esophagoscopy, neck ultrasonography (with or without fine needle aspiration cytology), head and neck contrast-enhanced computerized tomography and/or magnetic resonance imaging, chest X-rays, and liver ultrasonography.

Seventy-six patients had a primary partial laryngectomy $\left(28 \mathrm{CO}_{2}\right.$ laser-assisted excisions, 20 supraglottic laryngectomies, and 28 supracricoid laryngectomies) and 27 had a primary total laryngectomy (all performed at the Otolaryngology Section of Padova University). Eighty patients also had unilateral or bilateral cervical lymph node dissection, and postoperative radiotherapy (RT) was administered in 21 cases. The indications for neck dissection were based on currently accepted protocols. ${ }^{8}$ Postoperative RT was indicated in cases with one or more of the following adverse features: close/positive surgical margins; pT4 disease; perineural/lymphatic/vascular invasion; multiple positive nodes (three or more metastatic lymph nodes); extracapsular spread or perineural involvement; N3 nodes; subglottic extension of primary carcinoma. ${ }^{8}$ Based on the TNM classification of the Union Internationale Contre le Cancer (UICC) and the American Joint Committee on Cancer (AJCC) (sixth edition) ${ }^{9}$, the pathological staging of the primary laryngeal lesions (pT) was T1 in 34 cases, T2 in 34, T3 in 24, and T4a in 11; for the regional lymph nodes $(\mathrm{pN})$, it was $\mathrm{N}_{0}$ in 59 cases, $\mathrm{N} 1$ in 5 , and $\mathrm{N} 2$ in 16 . No distant metastases (M) were detected at diagnosis. As for pathological grading, 29 of the 103 cases were G1, 47 were G2, and 27 were G3. The mean follow-up was $42.2 \pm 22.6$ months.

All tissues were fixed in $4 \%$ para-formaldehyde and embedded in paraffin.

\section{Immunohistochemistry: mTOR}

Five- $\mu \mathrm{m}$ sections were cut from each tissue block for immunohistochemical examination after staining using a fully automated system (Bond-maX; Vision BioSystems, UK). Tissue sections were dewaxed and rehydrated by incubation at $72^{\circ} \mathrm{C}$ in Bond Dewax Solution (Vision BioSystems), 
ethanol, and distilled water. Antigens were retrieved by heating sections for 30 minutes at $100^{\circ} \mathrm{C}$ in Bond Epitope Retrieval Solution 1 (Vision BioSystems). Endogenous peroxidase was blocked with $3 \%$ hydrogen peroxide before 15 minutes of incubation with rabbit monoclonal anti-mTOR/FRAP, clone Y391(Novus Biologicals, Littleton, CO, USA), diluted 1:500. Specimens were then washed with phosphate-buffered saline $(\mathrm{pH} 7.0)$ and incubated with Bond Polymer Refine Detection Kit (Vision BioSystems) according to the manufacturer's protocols. The staining was visualized with 3,3'-diaminobenzidine (DAB) and the slides were counterstained with Mayer's hematoxylin. The sections were then dehydrated, cleared, and mounted. We used prostate cancer tissue as a positive mTOR control, while the primary antibody was substituted with phosphate-buffered solution for the negative control.

Image analysis (IA) mTOR determinations

All evaluations were performed on an image analysis workstation consisting of a conventional light Zeiss Axioskop microscope (Germany) with a color digital, Peltier-cooled videocamera (MicroPublisher 5.0 RTV) connected to a personal computer with the Image-Pro Plus Version $7^{\circledR}$ for Windows ${ }^{\mathrm{TM}}$ image analysis program (Media Cybernetics Inc, Bethesda, USA). In all cases, $1378 \times 954$ micron square areas of tumor tissue were evaluated with a 495-point sampling grid superimposed by the program on the image acquired with a $50 \mathrm{X}$ field of view (FOV). After counting the points that intercepted the positive and negative areas, the positive area fraction was calculated and reported as a percentage (\%).

To asses mTOR positive cases we empirically adopted a cut-off value of $13.1 \%$ corresponding to the median of the pooled mTOR expression in the present investigation. This cut-off value, that was analytically the best-fitting one, did not suffer of any subjectivity as it was a median value. This choice was supported also by the fact that no accepted cut-off values for mTOR expression in laryngeal SCC are available as yet.

Immunohistochemistry: c-myc, VEGF and p2 $7^{-i p 1}$ 
Five- $\mu$ m sections were cut from each tissue block of the 20 higher (mean $68.4 \% \pm 14.9 \%$, median $51.6 \%$ ) and 20 lower (mean $0.5 \% \pm 0.1 \%$, median $0.5 \%$ ) mTOR expression laryngeal SCCs. For each sample, c-myc reactivity (clone 9E11 mouse monoclonal antibody, diluted 1:100 [UCS Diagnostics srl, Roma, Italy]), VEGF reactivity (A-20:sc-152 rabbit polyclonal antibody, diluted 1:250 [Santa Cruz Biotechnology, Santa Cruz, CA]), and p27 ${ }^{\text {Kip1 }}$ reactivity (clone $1 \mathrm{~B} 4$ mouse monoclonal antibody, diluted 1:20 [Novocastra, Newcastle Upon Tyne, UK]) were evaluated. The positive controls were human breast carcinoma tissue for c-myc and VEGF, and tonsillar tissue for p2 $7^{\text {Kip1 }}$. The primary antibodies were substituted with phosphate-buffered solution for the negative controls. C-myc and VEGF immunoreactivity were classified according to immunostaining intensity into semi-quantitative scores from 0 to $3+(0$ : absent; $1+$ : low; $2+$ : medium; $3+$ : high). A minimum of 10 high power non-overlapping fields were evaluated for each case. $\mathrm{p} 27^{\mathrm{Kip} 1}$ was measured as a percentage of nuclear positivity. A minimum of 300 nuclei were counted at $20 x$ magnification.

\section{Statistical analysis}

The statistical tests used, as applicable, were Student's t-test, Fisher's exact test, the MannWhitney test, and the non-parametric test for trend (Kruskal-Wallis modification). The Kaplan-Meier product limit estimator and the log-rank test (to compare survival times stratified by $\mathrm{pT}$, N, stage, and mTOR expression) were also applied.

The receiver operating curve (ROC) approach (failure versus parameter) was adopted to set the analytically best-fitting cut-off value to binaries the continuous variable mTOR expression in the subgroup of mTOR-positive cases according to the highest level of the positive likelihood ratio.

In the multivariate analysis, Cox proportional hazard regression determined significant predictors of disease-free survival (DFS).

Two-sample Wilcoxon rank-sum test was applied to compare c-myc, VEGF and p27 $7^{\text {Kip1 }}$ immunoreactivity between the group of 20 patients with higher mTOR expression and the group of 20 patients with lower one. 
A $p$-value $<0.05$ was considered significant, while values in the range of $0.10>p \geq 0.05$ were considered as indicating a statistical trend. The STATATM 8.1 (Stata Corp, College Station, TX, USA) statistical package was used for all evaluations. 


\section{RESULTS}

Clinical outcome and follow-up (Table 1)

Seventy-two of the 103 cases of laryngeal SCC experienced no recurrent disease after treatment, while 31 developed loco-regional recurrences after a mean $14.3 \pm 10.5$ months. The mean follow-up was $43.2 \pm 23.0$ months for recurrence-free patients and $39.9 \pm 21.8$ months for those with recurrent disease (two-tailed t-test, $\mathrm{p}=0.49$ ). Fisher's exact test identified significant differences in the distributions for $p T(p=0.030)$, lymph node status $\left(N_{0} / N+\right)(p=0.006)$, and stage $(p=0.0001)$, but not for grade $(p=0.43)$, in the two subgroups of patients with and without loco-regional carcinoma recurrences. The log-rank test showed a significant difference in DFS (in months) when patients were stratified by $p T, N$ (Fig. 1$)$, and stage ( $p=0.049,0.0001$, and 0.0001 , respectively). Statistical analysis failed to disclose any significant difference in DFS when patients were stratified by grade (log-rank test, $\mathrm{p}=0.46)$.

The loco-regional recurrence rate was significantly lower in patients who had partial laryngectomy than in cases of total laryngectomy (Fisher's exact test, $p=0.007$ ), and DFS was significantly longer in the former (log-rank test, $p=0.003$ ). The recurrence rate was higher in patients given postoperative RT (Fisher's exact test, $p=0.015$ ), and their DFS was also significantly shorter (log-rank test, $p=0.0009$ ).

\section{mTOR-positive cases and clinico-pathological features}

Squamous cells of normal laryngeal mucosa were not stained by monoclonal antibody anti-mTOR (Fig. 2 A). Classical clinico-pathological parameters and mTOR expression in the 103 consecutive cases of laryngeal SCC have been synthesized in Table 1.

The mTOR expression in mTOR-negative laryngeal SCCs ranged from $0.1 \%$ to $13.1 \%$. Fifty-one cases of laryngeal SCC were mTOR-positive, with an expression $>13.1 \%$. Finely cytoplasmic granular staining was seen in the mTOR-positive laryngeal carcinoma cells (Fig. 2). The mTOR expression in these positive cases ranged from $14.2 \%$ to $85.0 \%$. (Table 2 ) 
In the sub-cohort of mTOR-positive cases, statistical analysis failed to disclose any significant differences in mTOR expression when these patients were distributed by $\mathrm{pT}$ (test for trend, $\mathrm{p}=0.82$ ), $\mathrm{N}$ (Mann Whitney U-test, $\mathrm{p}=0.87$ ), stage (test for trend, $\mathrm{p}=0.62$ ), or grade (test for trend, $\mathrm{p}=0.31$ ).

mTOR-positive cases and prognosis in laryngeal SCC

In the mTOR-positive patients, the ROC approach was used to find the analytically best-fitting cutoff for mTOR expression for prognostic purposes, and the value calculated was $50.7 \%$. Fifteen of the 51 mTOR-positive cases had an mTOR expression $>50.7 \%$. The loco-regional recurrence rate was significantly higher in laryngeal SCC patients with an mTOR expression $>50.7 \%$ (Fisher's exact test, $p=0.048$ ) (Table 2), and DFS was significantly shorter in these cases than in those with an mTOR expression $\leq 50.7 \%$ (log-rank test, $p=0.031$ ). (Fig $3 A$ ) When $\mathrm{N}_{0}$ patients were considered separately, statistical analysis showed a significantly higher disease recurrence rate in the group with an mTOR expression $>50.7 \%$ (Fisher's exact test, $\mathrm{p}=0.034$ ), and their DFS was also significantly shorter (log-rank test, $\mathrm{p}=0.009)$. (Fig 3B)

\section{mTOR-positive cases: multivariate analysis}

Multivariate DFS estimates were based on the Cox proportional hazards model and assumed no interactions between significant variables in the final model. Considering the sub-cohort of mTORpositive patients, mTOR status $(13.1 \%<$ mTOR expression $\leq 50.7 \%$ or $\mathrm{mTOR}$ expression $>50.7 \%)$, age, pT-stage, $\mathrm{N}$-stage, and grading were entered into this multivariate model to determine their relation with DFS. On analysis seen in Table 3, N-stage (risk ratio 42.2, 95\% confidence interval 7.2-274.0, $\mathrm{p}=0.0001$ ) and mTOR status (risk ratio $3.6,95 \%$ confidence interval $1.0-12.5, p=0.042$ ) maintained prognostic significance with relation to DFS.

\section{m-TOR and c-myc immunoreactivity (Fig 4A)}

In the 20 cases with lower mTOR expression, c-myc immunoreactivity was scored as 0 in 3 cases, as $1+$ in 7 , as $2+$ in 7 , as $3+$ in 2 . In one case c-myc immunoreactivity was not determinable. On 
the other hand, in the 20 cases with higher mTOR expression, c-myc immunoreactivity was scored as $1+$ in 2 cases, as $2+$ in 5 , as $3+$ in 11 . C-myc immunoreactivity was not determinable in 2 cases. Statistical analysis found a significantly higher c-myc immunoreactivity in the group of patients with higher mTOR expression (Wilcoxon rank-sum test, $\mathrm{p}=0.001$ ).

\section{m-TOR and VEGF immunoreactivity (Fig 4B)}

In the group of cases with lower mTOR expression, VEGF staining intensity was scored as $1+$ in 7 cases, as $2+$ in 7 , as $3+$ in 5 , undetectable in 1 . In the group of cases with higher mTOR expression, VEGF staining intensity was staged as $1+$ in 3 samples, as $2+$ in 4 , as $3+$ in 11 , undetectable in 2. VEGF immunoreactivity was significantly higher in samples with higher mTOR expression (Wilcoxon rank-sum test, $\mathrm{p}=0.02$ ).

$$
m-T O R \text { and } p 2 T^{K i p 1} \text { expressions. }
$$

Considering SCC specimens, neoplastic cells exhibited p2 $7^{\text {Kip1 }}$ staining confined to the nuclei (Fig 4C). The mean $\mathrm{p} 27^{\mathrm{Kip} 1}$ expressions were $47.1 \% \pm 31.6 \%$ (median $55.0 \%$ ) and $45.8 \% \pm 38.4 \%$ (median 40\%) in the groups of cases with lower and higher mTOR expressions, respectively (Wilcoxon rank-sum test, $\mathrm{p}=0.89$ ). 


\section{DISCUSSION}

In our series of consecutive laryngeal SCCs that underwent primary surgical treatment, statistical analysis found a significantly higher recurrence rate and shorter DFS in patients with advanced pathological stages according to the UICC/AJCC TNM classification. ${ }^{9}$ As expected, patients who had primary total laryngectomy for advanced SCC and those given postoperative RT had a worse prognosis. Cervical lymph node involvement is generally considered the most significant clinical prognostic factor in laryngeal $\mathrm{SCC}^{10}$, and N-stage correlated strongly with laryngeal SCC prognosis (in terms of disease recurrence rate, or DFS) in the present cohort too. Moreover, in our series multivariate analysis found that the relative risk of poor DFS with positive neck lymph nodes was 45.2.

Despite advances in the diagnosis and treatment of laryngeal SCC, patient survival has not improved and may even have deteriorated in the last two decades. ${ }^{11}$ There is an undeniable need for novel, more effective therapeutic strategies to improve overall survival for patients with laryngeal SCC, and for cases with advanced disease in particular. One promising approach is receptor-mediated carcinoma-targeted therapy.

Although several previous studies investigated the biological role of mTOR in carcinogenesis and development of head and neck SCC considering in particular carcinoma cell lines or anonymous head and neck SCC tissues ${ }^{12-15}$, there are still no data correlating mTOR expression with outcome in laryngeal SCC patients. In the present study, we investigated only surgical specimens (not biopsies) of carcinoma and only from larynxes treated primarily and consecutively by the same surgical team in order to reduce the risk of a significant bias due to the heterogeneity typical of retrospective series of head and neck SCCs. For immunohistochemical investigations, we used a computer-based image analysis system for its accuracy, precision and reproducibility in immunostained slide analysis. We found the intensity of staining in our samples very weak by comparison with the findings in prostate cancer, which served as the positive control for mTOR. Since there is no validated cut-off for mTOR immunohistochemical positivity in laryngeal SCC, the median value of the pooled mTOR expression levels $(13.1 \%)$ was chosen as the cut-off 
for the present study, since it does not suffer from any subjective considerations or post-hoc construction.

Fifty-one cases of laryngeal SCC were mTOR-positive, with an expression $>13.1 \%$. Cytoplasmic staining was determined in the mTOR-positive laryngeal carcinoma cells. No significant associations emerged in our mTOR-positive patients between mTOR expression and the conventional clinico-pathological parameters ( $\mathrm{pT}, \mathrm{N}$, stage, grade). Patients with an mTOR expression $>50.7 \%$ had a significantly higher loco-regional laryngeal SCC recurrence rate, however, and statistical analysis showed that they had a significantly shorter DFS than in cases with an mTOR expression $\leq 50.7 \%$. Moreover, when $\mathrm{N}_{0}$ patients were considered separately, the disease recurrence rate was found higher and the DFS shorter for the mTOR-positive patients who had an mTOR expression $>50.7 \%$. Multivariate analysis showed that $\mathrm{mTOR}$ expression $>50.7 \%$ had a prognostic significance with relation to DFS. In particular, it was noted that the relative risk of poor DFS with mTOR expression $>50.7 \%$ was 3.6. Furthermore, multivariate model evidence confirmed the hypothesis that mTOR high expression in carcinoma cells was an $\mathrm{N}$-stage independent indicator of a poor prognosis for laryngeal SCC. In the light of the above, mTOR expression should be further investigated as a promising prognostic indicator in laryngeal SCCs. Our preliminary results support the hypothesis that mTOR expression in laryngeal SCC may be useful for detecting patients at higher risk of disease recurrence after treatment, and $\mathrm{N}_{0}$ patients at higher risk of early loco-regional carcinoma recurrence who might benefit from more aggressive therapy. Further investigations considering larger series of laryngeal SCCs and, in particular, cohorts of patients with advanced, unresectable SCCs who underwent primary chemoradiotherapy are mandatory.

$\mathrm{m}$-TOR is a signaling molecule that integrates a broad spectrum of signals. We preliminarily investigated by immunohistochemistry the relation between mTOR and c-myc, VEGF and p27 ${ }^{\text {Kip1 }}$ expressions in the 20 patients with higher mTOR expression and in the 20 patients with lower mTOR expression. C-myc seems to play a pivotal role in cellular transformation and carcinogenesis. Over-expression of the c-myc oncogene has been observed in many solid tumors. The role of c-myc protein in head and neck SCC in general and laryngeal SCC in particular is far 
from clear. ${ }^{16}$ In the present study, we found a significantly higher c-myc immunoreactivity in the group of patients with higher mTOR expression. In human hepatocellular carcinoma, PIK3CA/Akt/mTOR and c-myb/COX-2 pathways were similarly activated, with levels of PIK3CA/Akt, mTOR, and c-myb being inversely associated with patients' survival length. Knocking down c-myc and E2F1 oncoproteins reduced PIK3CA/Akt and mTOR and completely abolished cmyb and COX-2 expression in human hepatocellular carcinoma cell lines. ${ }^{17} \mathrm{~A}$ detailed metaanalysis of head and neck SCC patients found relative risk of death to be approximately 2-fold higher in VEGF-positive tumors. ${ }^{18}$ In our limited experience, VEGF immunoreactivity was significantly higher in laryngeal SCC samples characterized by higher mTOR expression. Mulders $^{19}$ recently stated that mTOR inhibitors suppress hypoxia-inducible factor-1a (HIF) transcription levels in tumor cells, thus reducing VEGF expression and renal cell carcinoma angiogenesis. p27 protein plays a negative role in cell-cycle progression from the $\mathrm{G} 1$ to the $\mathrm{S}$ phase and is considered a tumor suppressor. p27 down-regulation was demonstrated in several malignancies. Only a few studies investigated p27's potential prognostic role in laryngeal SCC. ${ }^{20}$ In 1999, Pruneri et al. $^{21}$ analyzed p27 expression in 132 consecutive cases of laryngeal SCC concluding that the worst prognosis was found in the small group of patients with very low p27 expression $(<5 \%)$. On the other hand, in a cohort of 59 patients (including 13 hypopharyngeal carcinomas), Liu et al. $^{22}$ did not find any statistical relationship between p27 expression and survival. In the present investigation, statistical analysis ruled out any significant difference between the mean p27 $7^{\text {Kip1 }}$ expressions of the 20 patients with higher mTOR expression and the 20 patients with lower mTOR expression.

The mTOR inhibitors, such as modern rapamycin analogues, which have a more favorable pharmacokinetic profile than the parent molecule, have been assessed in terms of their safety (phase I safety analyses showed that they are generally well tolerated ${ }^{23}$ ) and efficacy in patients with cancer. The most promising results obtained with mTOR inhibitors so far have been in renal cell carcinoma, mantle cell lymphoma and endometrial cancer ${ }^{24}$, and have prompted extensive clinical programs to evaluate mTOR inhibitors more thoroughly in several tumor types. Pivotal clinical trials with mTOR inhibitors in neuroendocrine tumors, pancreatic islet cell tumors, breast 
cancer, hepatocellular carcinoma, and gastric cancer are currently underway. ${ }^{23}$ Targeted therapies for the organ-preserving treatment of advanced laryngeal SCC are now attracting a great deal of attention and, very recently, Lei et al. ${ }^{25}$ was the first to culture Hep-2 laryngeal cancer cells in the presence of different concentrations of rapamycin, cisplatin, or both. A preliminary assessment was conducted on cell growth inhibition, apoptosis, and AKT, mTOR, S6K and ERCC1 protein levels. All combinations of rapamycin and cisplatin induced a synergistic cell growth inhibition. Rapamycin inhibited Hep-2 cell growth, induced G1 arrest and, when combined with cisplatin, enhanced apoptosis. Ekshyyan et al. ${ }^{26}$ evaluated the effects of temsirolimus, a rapamycin-analog, on Akt/mTOR pathway biomarkers in tumor and peripheral blood mononuclear cells from 16 patients with advanced head and neck (oral/oropharyngeal) SCCs receiving chemoradiotherapy. Only 10 patients completed all 3 planned doses of temsirolimus and underwent post-treatment biopsy, which revealed significant reductions of pS6 and 4E-binding protein 1 in tumor tissue. The related side effects were reportedly minimal and reversible. The value of the Ekshyyan et al. ${ }^{26}$ clinical trial is extremely limited, of course, but temsirolimus seems promising for mTOR pathway inhibition in patients with head and neck SCC.

Further investigations are naturally needed to establish the future prospects of incorporating modern mTOR inhibitors in multimodality or multi-target strategies (for example including a VEGF directed monoclonal antibody) against laryngeal SCC. 


\section{REFERENCES}

1. Mita MM, Mita A, Rowinsky EK. The molecular target of rapamycin (mTOR) as a therapeutical target against cancer. Cancer Biol Ther 2003;2:S169-S177.

2. Dancey JE. Therapeutic targets: MTOR and related pathways. Cancer Biol Ther 2006;5:1065-1073.

3. Hudes GR. Targeting mTOR in renal cell carcinoma. Cancer 2009;115 (Suppl 10):23132320.

4. Yu G, Wang J, Chen Y, et al. Overexpression of phosphorylated mammalian target of rapamycin predicts lymph node metastasis and prognosis of Chinese patients with gastric cancer. Clin Cancer Res 2009;15:1821-1829.

5. Rowinsky EK. Targeting the molecular target of rapamycin (mTOR). Curr Opinion Oncol 2004;16:564-575.

6. Jiang BH, Liu LZ. Role of mTOR in anticancer drug resistance: perspectives for improved drug treatment. Drug Resist Updat 2008;11:63-76.

7. No JH, Jeon YT, Park IA, et al. Expression of mTOR protein and its clinical significance in endometrial cancer. Med Sci Monit 2009;15:301-305.

8. Marioni G, Marchese-Ragona R, Cartei G, Marchese F, Staffieri A. Current opinion in diagnosis and treatment of laryngeal carcinoma. Cancer Treatment Reviews 2006;32:504-515.

9. Greene FL, Page DL, Fleming ID, et al., eds. AJCC Cancer Staging Manual. 6th ed. New York, NY: Springer; 2002.

10. Pinsolle J, Pinsolle V, Majoufre C, Duroux S, Demeaux H, Siberchicot F. Prognostic value of histologic findings in neck dissections for squamous cell carcinoma. Arch Otolaryngol Head Neck Surg 1997;123:145-148.

11. Hoffman HT, Porter K, Karnell LH, et al. Laryngeal cancer in the United States: changes in demographics, patterns of care, and survival. Laryngoscope 2006;116 (Suppl 111):1-13. 
12. Amornphimoltham $\mathrm{P}$, Patel $\mathrm{V}$, Sodhi $\mathrm{A}$, et al. Mammalian target of rapamycin, a molecular target in squamous cell carcinomas of the head and neck. Cancer Cell Res 2005;65:9953-9961.

13. Liu FY, Zhao ZJ, Li P, Ding X, Zong ZH, Sun CF. Mammalian target of rapamycin (mTOR) is involved in the survival of cells mediated by chemokine receptor 7 through PI3K/Akt in metastatic squamous cell carcinoma of the head and neck. Br J Oral Maxillofac Surg 2010;48:291296.

14. Molinolo AA, Hewitt SM, Amornphimoltham $P$, et al. Dissecting the Akt/mammalian target of rapamycin signaling network: emerging results from the head and neck cancer tissue array initiative. Clin Cancer Res 2007;13:4964-4973.

15. Nathan CA, Amirghahari N, Rong X, et al. Mammalian target of rapamycin inhibitors as possible adjuvant therapy for microscopic residual disease in head and neck squamous cell cancer. Cancer Res 2007;67:2160-2168.

16. Krecicki T, Fraczek M, Jelen M, Zatonski T, Szkudlarek T, Dus D. Expression of c-myc oncoprotein in laryngeal squamous cell carcinoma. Acta Otolaryngol (Stockh) 2004;124:634-637.

17. Ladu S, Calvisi DF, Conner EA, Farina M, Factor VM, Thorgeirsson SS. E2F1 inhibits cMyc-driven apoptosis via PIK3CA/Akt/mTOR and COX-2 in a mouse model of human liver cancer. Gastroenterology 2008;135:1322-1332.

18. Kyzas PA, Cunha IW, loannidis JP. Prognostic significance of vascular endothelial growth factor immunohistochemical expression in head and neck squamous cell carcinoma: a metaanalysis. Clin Cancer Res 2005;11:1434-1440.

19. Mulders P. Vascular endothelial growth factor and mTOR pathways in renal cell carcinoma: differences and synergies of two targeted mechanisms. BJU Int 2009;104:1585-1589.

20. Calgaro N, Marioni G, Blandamura S, et al. P27 and MIB-1 expression is related to malignancy recurrence in laryngeal carcinoma treated with partial laryngectomy: preliminary results. J Otolaryngol 2007;36:98-105. 
21. Pruneri G, Pignataro L, Carboni N, et al. Clinical relevance of expression of the CIP/KIP cell-cycle inhibitors p21 and p27 in laryngeal cancer. J Clin Oncol 1999;17:3150-3159.

22. Liu M, Lawson G, Delos M, et al. Prognostic value of cell proliferation markers, tumour suppressor proteins and cell adhesion molecules in primary squamous cell carcinoma of the larynx and hypopharynx. Eur Arch Otorhinolaryngol 2003;260:28-34.

23. Yuan R, Kay A, Berg WJ, Lebwohl D. Targeting tumorigenesis: development and use of mTOR inhibitors in cancer therapy. J Hematol Oncol 2009;2:45.

24. Faivre S, Kroemer G, Raymond E. Current development of mTOR inhibitors as anticancer agents. Nature Reviews 2006;5:671-688.

25. Lei W, Jia T, Su Z, Wen W, Zhu X. Combined effect of rapamycin and cisplatin on survival of Hep-2 cells in vitro. Oncol Res 2009;18:73-81.

26. Ekshyyan O, Mills GM, Lian T, et al. Pharmacodynamic evaluation of temsirolimus in patients with newly diagnosed advanced-stage head and neck squamous cell carcinoma. Head Neck, in press 


\section{LEGEND TO FIGURES}

Fig 1. Disease-free survival estimates by $\mathrm{N}$-stage $\left(\mathrm{N}_{0}\right.$ vs $\left.\mathrm{N}_{+}\right)$in 103 consecutive laryngeal SCCs; time calculated in months.

Fig. 2. mTOR expression (rabbit monoclonal anti-mTOR/FRAP, clone Y391 [Novus Biologicals, Littleton, CO, USA], diluted 1:500) in normal laryngeal mucosa and in laryngeal SCC cells.

Fig. 3. A. Disease-free survival estimates by mTOR expression in mTOR-positive laryngeal SCCs (51 cases) [13.1\%< mTOR expression $\leq 50.7 \%$ or $>50.7 \%$; time calculated in months; $\mathrm{B}$. Disease-free survival estimates in $45 \mathrm{~N}_{0}$ mTOR-positive patients with laryngeal SCC [13.1\%< mTOR expression $\leq 50.7 \%$ or $>50.7 \%$; time calculated in months.

Fig 4. A. c-myc staining in laryngeal SCC; B. VEGF staining in laryngeal SCC; C. p27 $7^{\text {Kip1 }}$ staining in laryngeal SCC. 
Table 1. Classical clinico-pathological parameters, prognosis and mTOR expression in 103 consecutive cases of laryngeal SCC

\begin{tabular}{|c|c|c|c|c|}
\hline & No. of cases & $\begin{array}{l}\text { No. of cases } \\
\text { with loco-regional } \\
\text { recurrence }\end{array}$ & $\begin{array}{c}\text { Mean } \\
\text { disease-free survival } \\
\text { (in months) } \\
\pm \mathrm{SD}\end{array}$ & $\begin{array}{c}\text { Mean } \\
\text { mTOR expression } \pm S D\end{array}$ \\
\hline pT1 & 34 & 5 & $42.9 \pm 24.6$ & $24.3 \pm 23.1$ \\
\hline pT2 & 34 & 15 & $33.0 \pm 25.6$ & $25.4 \pm 27.3$ \\
\hline pT3 & 24 & 6 & $29.8 \pm 20.8$ & $18.3 \pm 27.2$ \\
\hline pT4a & 11 & 5 & $23.2 \pm 17.5$ & $17.0 \pm 25.5$ \\
\hline $\mathrm{N}_{0}\left(\mathrm{cN}_{0}+\mathrm{pN}_{0}\right)$ & 82 & 19 & $38.5 \pm 24.2$ & $24.2 \pm 26.1$ \\
\hline $\mathrm{N}+$ & 21 & 12 & $18.9 \pm 16.3$ & $15.1 \pm 23.0$ \\
\hline Stage I & 34 & 5 & $42.9 \pm 24.6$ & $24.3+23.2$ \\
\hline Stage II & 26 & 12 & $35.5 \pm 26.9$ & $28.8 \pm 25.8$ \\
\hline Stage III & 19 & 1 & $38.5 \pm 20.4$ & $19.4 \pm 29.8$ \\
\hline Stage IV & 24 & 13 & $18.3 \pm 13.8$ & $17.7 \pm 25.8$ \\
\hline G1 & 29 & 6 & $424+259$ & $292+270$ \\
\hline G2 & 47 & 16 & $30.7 \pm 21.3$ & $20.2 \pm 23.8$ \\
\hline G3 & 27 & 9 & $32.5 \pm 25.4$ & $19.3 \pm 27.0$ \\
\hline Partial laryngectomy & 76 & 17 & $38.6 \pm 23.9$ & \\
\hline Total laryngectomy & 27 & 14 & $22.9 \pm 21.0$ & \\
\hline & & & & \\
\hline Postoperative RT & 21 & 11 & $23.1 \pm 21.7$ & \\
\hline No postoperative RT & 82 & 20 & $37.4 \pm 23.9$ & \\
\hline
\end{tabular}


Table 2. Classical clinico-pathological and prognostic parameters and mTOR expression in 51 mTORpositive cases of laryngeal SCC

\begin{tabular}{|c|c|c|}
\hline & $\begin{array}{l}\text { No. of } \\
\text { cases }\end{array}$ & $\begin{array}{c}\text { Mean } \\
\text { mTOR expression } \pm S D\end{array}$ \\
\hline pT1 & 20 & $37.4 \pm 21.8$ \\
\hline pT2 & 17 & $46.8 \pm 23.4$ \\
\hline pT3 & 11 & $37.9 \pm 30.2$ \\
\hline pT4 & 3 & $50.7 \pm 28.6$ \\
\hline $\mathrm{N}_{0}\left(\mathrm{cN}_{0}+\mathrm{pN}_{0}\right)$ & 45 & $41.1 \pm 24.5$ \\
\hline $\mathrm{N}+$ & 6 & $43.9 \pm 25.5$ \\
\hline Stage I & 20 & $37.4+21.8$ \\
\hline Stage II & 15 & $43.1 \pm 22.4$ \\
\hline Stage III & 8 & $43.0 \pm 34.1$ \\
\hline Stage IV & 8 & $46.5 \pm 26.6$ \\
\hline G1 & 2 & $402+254$ \\
\hline G2 & 21 & $39.6 \pm 23.8$ \\
\hline G3 & 10 & $47.7 \pm 25.0$ \\
\hline E & & \\
\hline Partial laryngectomy & 37 & $41.1 \pm 23.2$ \\
\hline Total laryngectomy & 14 & $42.2 \pm 28.3$ \\
\hline & & \\
\hline Postoperative RT & 9 & $41.3 \pm 24.4$ \\
\hline No postoperative RT & 42 & $41.9 \pm 25.6$ \\
\hline $\begin{array}{l}\text { With loco-regional } \\
\text { recurrence }\end{array}$ & 14 & $49.3 \pm 24.5$ \\
\hline $\begin{array}{l}\text { Without loco-regional } \\
\text { recurrence }\end{array}$ & 37 & $38.4 \pm 24.0$ \\
\hline
\end{tabular}


Table 3. Multivariate analysis of prognostic factors for disease-free survival (DFS) in mTOR-positive patients

\begin{tabular}{|c|c|c|c|}
\hline Prognostic factors & Risk ratio & 95\% confidence intervals & $p$ \\
\hline \multicolumn{4}{|l|}{ Age (in years) } \\
\hline$<60$ & 1.0 & Reference group & 0.91 \\
\hline$\geq 60$ & 3.1 & $0.5-18.4$ & \\
\hline \multicolumn{4}{|l|}{ pT-stage } \\
\hline pT1-T2 & 1.0 & Reference group & 0.15 \\
\hline pT3-T4 & 0.3 & $0.03-1.2$ & \\
\hline \multirow{2}{*}{\multicolumn{4}{|c|}{$\mathrm{N}$-stage }} \\
\hline & & & \\
\hline $\mathrm{N} 0\left(\mathrm{cN}_{0}+\mathrm{pN}_{0}\right)$ & 1.0 & Reference group & 0.0001 \\
\hline $\mathrm{N}+$ & 45.2 & $7.2-274.0$ & \\
\hline \multicolumn{4}{|l|}{ Grading (G) } \\
\hline G1-G2 & 1.0 & Reference group & 0.87 \\
\hline G3 & 0.9 & $0.2-4.7$ & \\
\hline \multicolumn{4}{|l|}{ mTOR status } \\
\hline $13.1 \%<$ mTOR expression $\leq 50.7 \%$ & 1.0 & Reference group & 0.042 \\
\hline mTOR expression $>50.7 \%$ & 3.6 & $1.0-12.5$ & \\
\hline
\end{tabular}




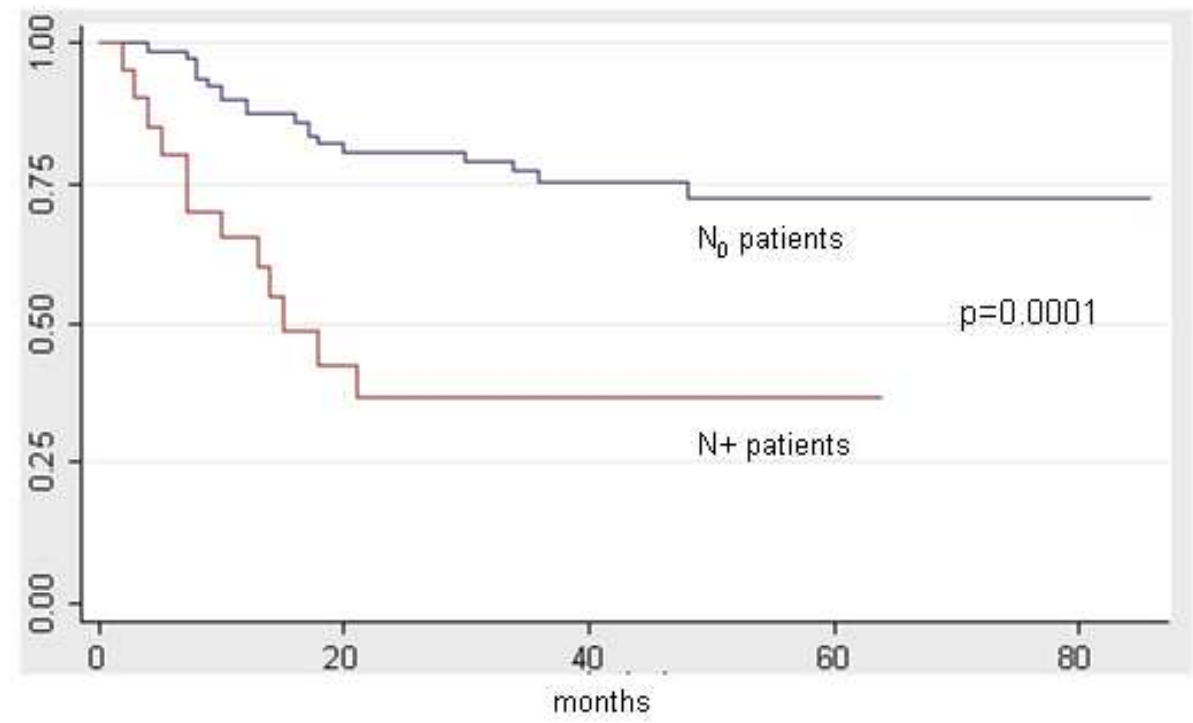

$150 \times 119 \mathrm{~mm}(96 \times 96 \mathrm{DPI})$ 


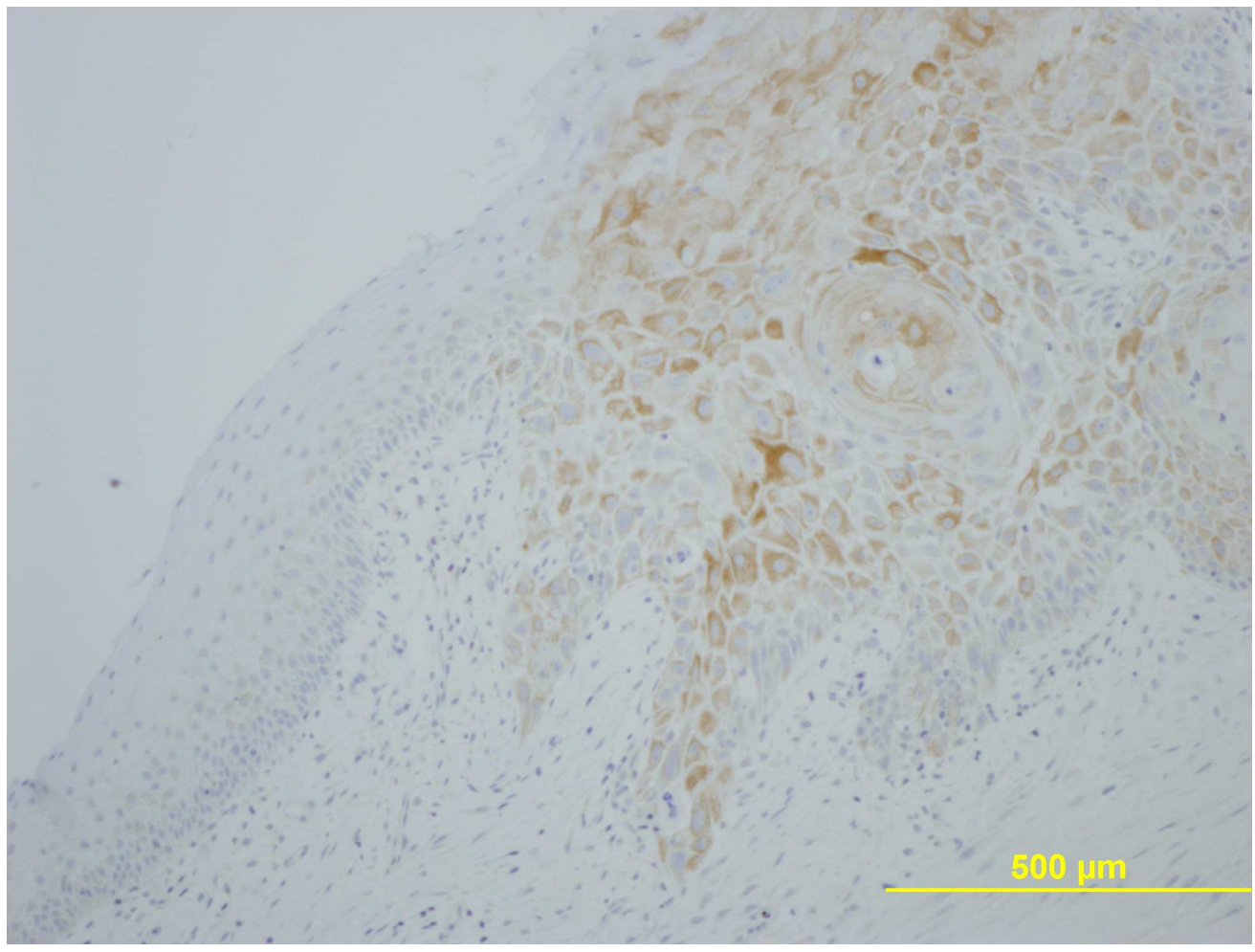

$539 \times 406 \mathrm{~mm}(96 \times 96$ DPI) 


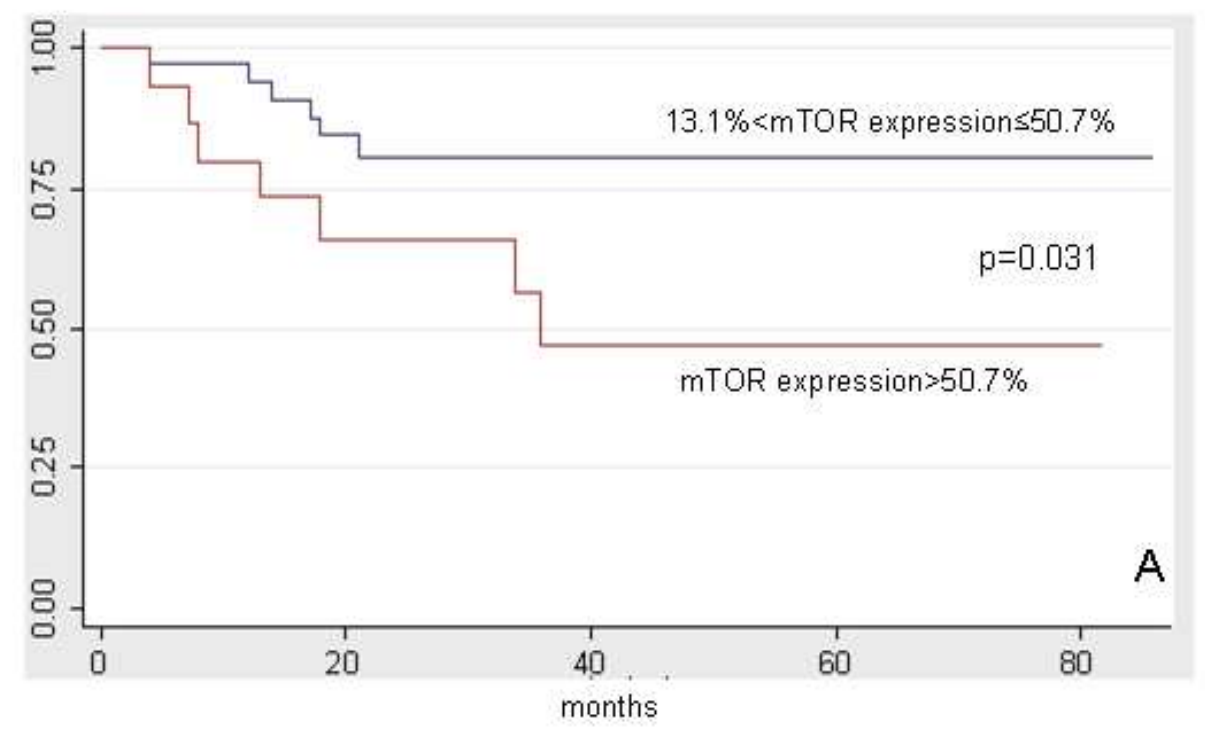

$150 \times 119 \mathrm{~mm}(96 \times 96$ DPI) 


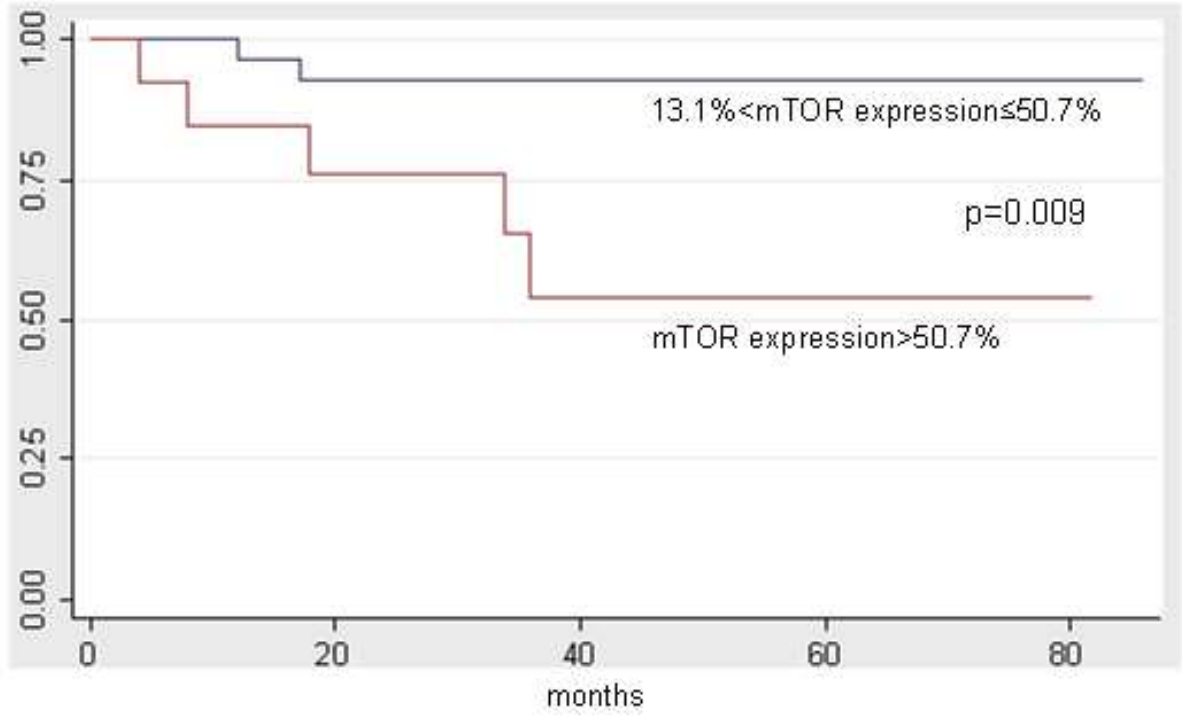

$150 \times 119 \mathrm{~mm}(96 \times 96$ DPI) 

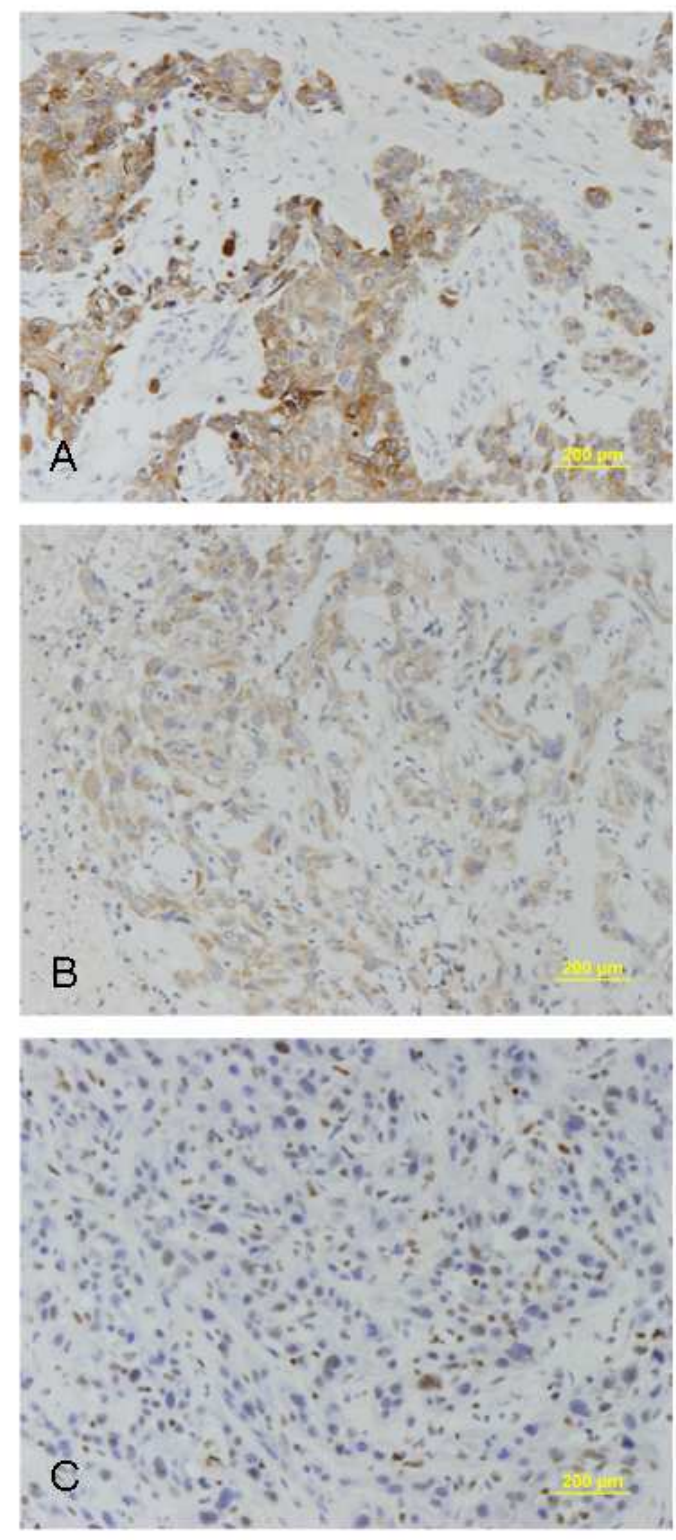

$129 \times 229 m m(96 \times 96$ DPI $)$ 


\section{DIPARTIMENTO DI SPECIALITA'MEDICO-CHIRURGICHE CLINICA OTORINOLARINGOIATRICA}

Direttore: Prof. A. Staffieri

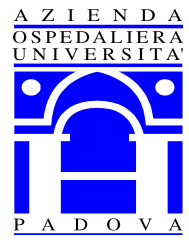

Editor-in-Chief

Histopathology

Dear Editor,

Padova, March 20, 2010

We are sending you the manuscript "MAMMALIAN TARGET OF RAPAMYCIN (mTOR)

EXPRESSION AND LARYNGEAL SQUAMOUS CELL CARCINOMA PROGNOSIS:

NOVEL PRELIMINARY EVIDENCE" which we would like you to consider for publication in

Histopathology.

CONFLICT OF INTEREST STATEMENT

The authors of the paper understand and fully accept the policies of the Journal.

We declare NO financial and personal relationships with other people or organisations that could inappropriately influence (bias) our work.

THE PRESENT PAPER IS ORIGINAL AND HAS NOT BEEN AND WILL NOT BE PUBLISHED, IN PART OR IN WHOLE, IN ANY OTHER JOURNAL

ALL AUTHORS HAVE CONTRIBUTED SUFFICIENTLY, APPROVED THE

MANUSCRIPT AND ITS SUBMISSION TO Histopathology.

Best regards

Gino Marioni, MD

Correspondence to:

Gino Marioni, MD

Dept Medical and Surgical Specialties, Otolaryngology Section, University of Padova

Via Giustiniani 2, 35100 Padova, Italy

gino.marioni@unipd.it

fax $+39(0) 498213113$ 